\title{
Phénomènes d'électrisation des matériaux isolants pour transformateurs de puissance
}

\author{
L. Peyraque $\left({ }^{1}\right)$, A. Béroual $\left({ }^{2}\right)$, C. Boisdon $\left({ }^{1}\right)$ et F. Buret $\left({ }^{2}\right)$ \\ (') Jeumont-Schneider Transformateurs, 84 avenue Paul Santy, 69371, Lyon Cedex 08, France \\ (2) Département d'Electrotechnique, CEGELY (URA 829 C.N.R.S.), Ecole Centrale de Lyon, \\ B.P. 163, 69131 Ecully Cedex, France
}

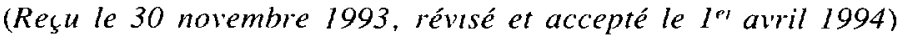

\begin{abstract}
Résumé. - Dans les transformateurs de puissance, la circulation d'huıle à travers les canaux de refroidissement en fibre cellulosique (carton isolant) est à l'origine d'une séparation de charges. L'étude de ce phénomène d'électrisation statique est présentée, tout d'abord à travers un dispositif permettant d'étudier la séparation de charges créées par le mouvement du carton dans l'huile. Celu1-ci a permis de mettre en évidence l'influence de plusieurs paramètres comme la vitesse, la température, l'humidité et le traitement des huiles et cartons ainsi que la nature du carton sur cette charge. Le second dispositif présenté permet de classer les huiles selon leur niveau de création de charges. Une corrélation semble s'établir entre la densité de ces charges et les grandeurs caractéristiques de l'huile telles que la conductivité, la rigidité diélectrique et la teneur en eau.

Abstract. - In power transformers, the oil flowed through the cooling ducts in coil assembly induces a static charge separation. The study of this static electrification phenomena is presented through a device allowing to investigate the charge separation generated by the rotating motion of a transformer pressboard in oil. This permits to show the influence of various parameters such as velocity, temperature, moisture and processing of oils and pressboards as well as their nature on this electrical charge. We also present another device allowing to classify oils according to their level of charge production. It appears from our results that a correlation exists between the density of these charges and the characteristics of oil such as conductivity, electric strength and water content.
\end{abstract}

\section{Introduction.}

L'apparition de nouveaux matériaux isolants. l'utilisation de techniques de purification de plus en plus sophistiquées permettant d'obtenir des huiles de très haute résistivité. ainsi que l'amélioration des méthodes de séchage pour minimiser l'humidification des matériaux cellulosiques en particulier, ont permis d'améliorer les niveaux d'isolement. L'augmentation des tensions et des puissances de transformateurs et la diminution de leurs gabarits, ont conduit à la mise en œuvre de systèmes de refroidissement par circulation d'huile à des débits de plus en plus importants. 
L'écoulement forcé de cette huile, ainsi que son contact avec les surfaces cellulosiques, sont à l'origine de la création de charges statiques. Celles-ci sont en effet produites grâce aux phénomènes de séparation, de migration et de relaxation de charges à l'interface solide/liquide $[1,2]$. Ainsi, une double couche électrique est créée par absorption de charges par le diélectrique solide. En général, le papier se charge négativement et l'huile positivement [3, 4]. Les propriétés physico-chimiques de l'interface solide/liquide, la vitesse d'écoulement, la température, la teneur en eau de l'huile, l'humidité du solide isolant et son état de surface (rugosité en particulier) sont autant de paramètres dont peut dépendre le degré d'électrisation.

L'objet de la présente étude est de mettre en évidence l'influence de certains de ces paramètres sur le mécanisme d'électrisation statique pour différents couples de matériaux solide/liquide utilisés dans l'isolation des transformateurs. Nous présentons également les techniques expérimentales permettant d'étudier les caractéristiques fondamentales de séparation de charges aux interfaces ainsi qu'un dispositif de mesure de la tendance à la charge des huiles lors de leurs passages à travers un filtre et pouvant servir de base à une comparaison entre les huiles.

\section{Techniques expérimentales.}

Deux dispositifs de mesure sont utilisés: l'un, basé sur une cellule d'essai dite « de type CIGRE », pour la mesure de la charge produite lors du contact huile/carton et l'autre pour apprécier la tendance à la charge d'une huile donnée.

2.1 Cellule D'essai « TYPE CIGRE ». - Elle est issue d'une synthèse entre les cellules de test de l'industrie pétrolière et celles déjà utilisées dans le passé par l'industrie électrotechnique $[5,6]$. Elle est constituée d'un disque tournant métallique recouvert sur ces deux faces du matériau solide à étudier (carton dans notre cas) et immergé dans une cuve d'huile d'essai où il peut être mis en mouvement grâce à un petit moteur électrique (Fig. 1). Le disque, de diamètre $150 \mathrm{~mm}$ et d'épaisseur $6 \mathrm{~mm}$, est placé au centre de la cuve de diamètre $250 \mathrm{~mm}$ et de hauteur $80 \mathrm{~mm}$.

Ce système, réalisé en aluminium, permet de quantifier la charge recueillie sur le disque. En effet, grâce à un électromètre, on peut mesurer le courant créé sur le disque et s'écoulant à la masse (Fig. 2). Celui-ci est mesuré sur une période de $30 \mathrm{~min}$, le courant transitoire apparaissant en début de rotation (durant les premières minutes de l'essai) n'est pas pris en considération. La cellule et l'appareil de mesure sont placés dans une cage de Faraday.

Nous utilisons deux types d'huiles neuves, l'une naphténique (huile H1), l'autre parafinique (huile $\mathrm{H} 2$ ) en association dans différentes configurations avec deux types de carton $\mathrm{C} 1$ et $\mathrm{C} 2$ d'épaisseur $2 \mathrm{~mm}$. C2 est un carton pré-compressé, plus dense, plus dur et dont la surface est moins lisse que celle de $\mathrm{C} 1$. La viscosité des huiles $\mathrm{H} 1$ et $\mathrm{H} 2$ à $20^{\circ} \mathrm{C}$ est comprise entre 17 et $20 \mathrm{cSt}$.

2.2 Dispositif De Mesure de LA TENDANCE À LA CHARge deS Huiles. - Pour compléter les mesures précédentes, nous utilisons un autre dispositif qui permet de connaître et de quantifier la tendance d'une huile à créer des charges au passage d'un filtre [7]. Ce système permet entre autre, de comparer les différentes huiles et de corréler leur tendance à la charge avec leurs propriétés diélectriques (tenue diélectrique, conductivité), leur teneur en eau et leur vieillissement.

Son principe consiste à forcer une huile, supposée électriquement neutre au départ, à travers un filtre cellulosique. Une séparation de charges s'opère alors. L'huile se charge positivement et le filtre négativement. Ainsi, on obtient la quantité de charges créée par écoulement, pour une unité de volume d'huile donnée. Si $Q$ est la charge, $V$ le volume d'huile écoulé et $I$ le 


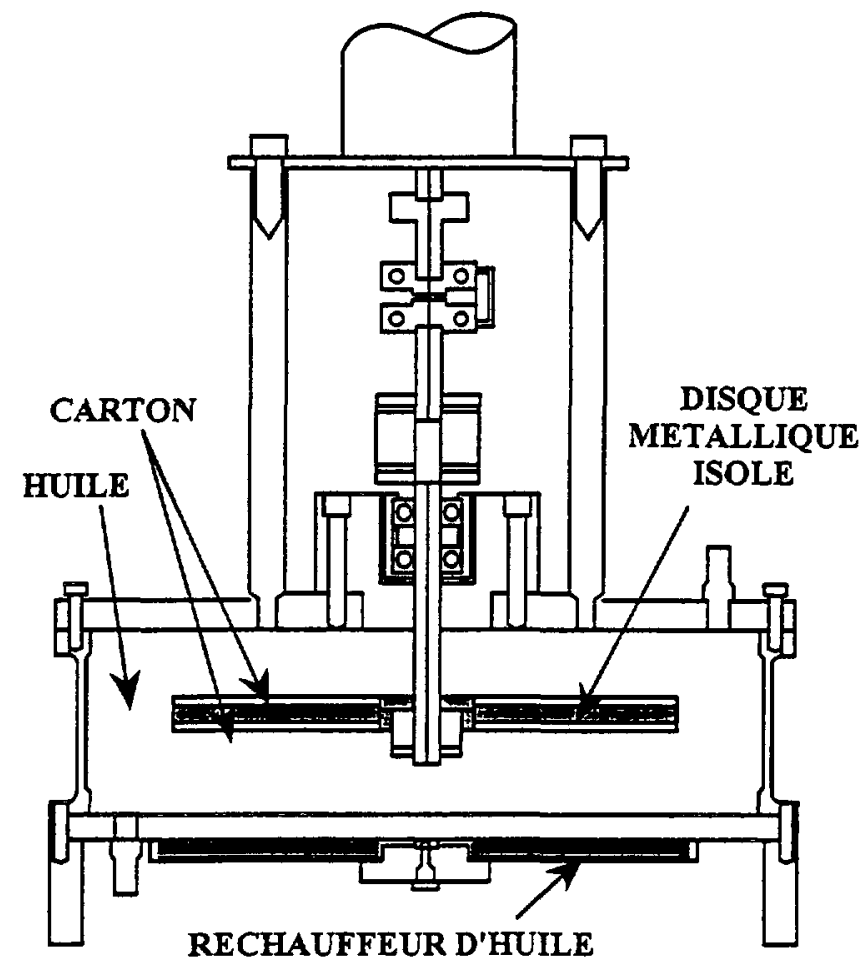

Fig. 1. - Cellule - type CIGRE - pour la mesure de l'électrisation statique d'un couple carton-huile. [Test cell - CIGRE type - for the static electrification measurement of pressboard-oil couple.]

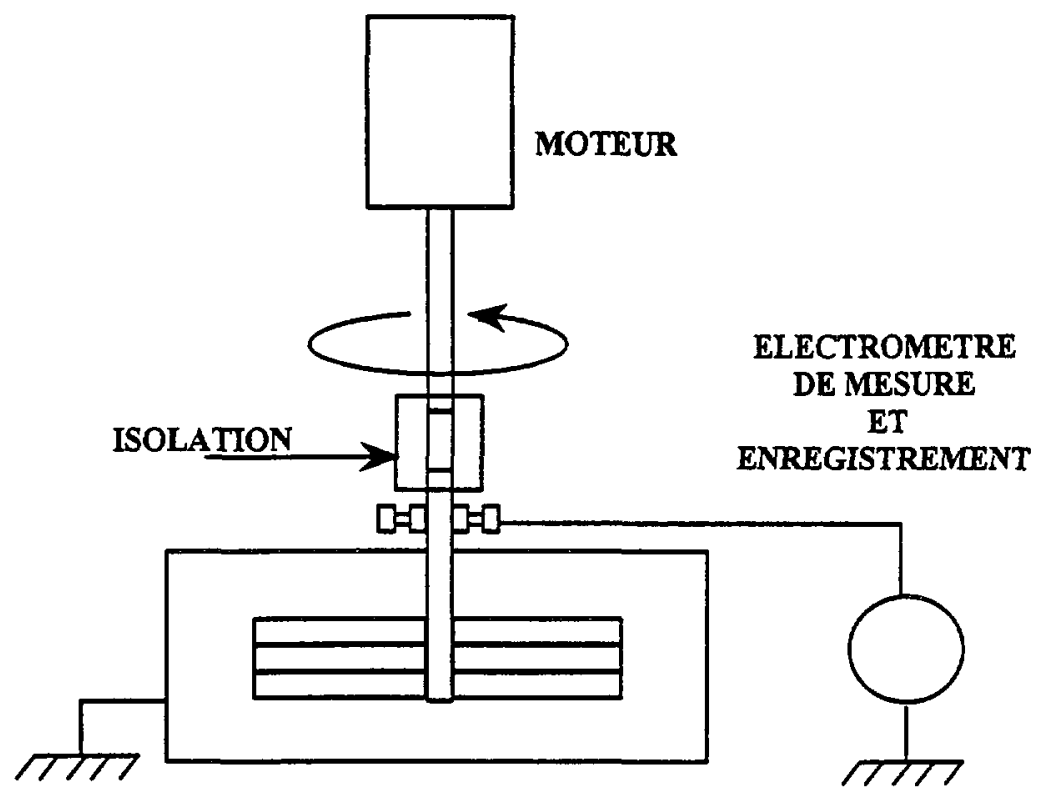

Fig. 2. - Dispositif de mesure des charges électrostatiques sur cellule CIGRE.

[Measuring system of electrostatic charges with CIGRE test cell.] 


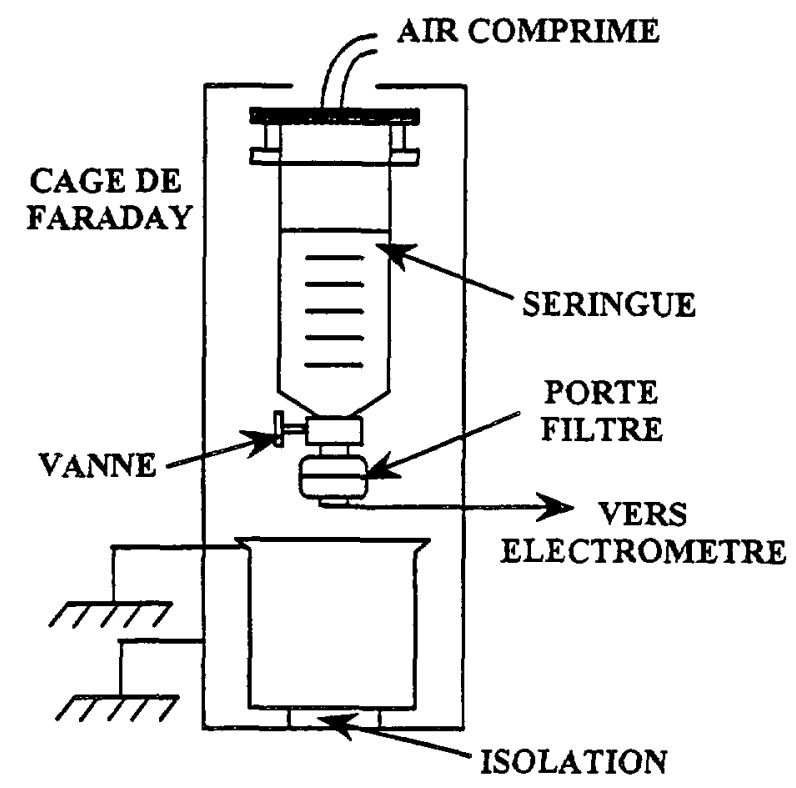

Fig. 3. - Description et principe de la mesure de la tendance à la charge des huiles.

[Description and principle of the measuring method of the electrostatic charging tendency of oils.]

courant moyen. la densité de charge $\rho$ est donnée par la relation :

$$
\rho=Q / V=I t / V=I /(V / t)=\text { courant de charge/débit d'huile } .
$$

Cette densité de charge est connue sous le terme anglais «Electrostatic Charging Tendency » (E.C.T.). Cependant, les mesures dépendent du type de filtre utilisé et des conditions de mesure. Cette mesure est effectuée grâce à une couronne métallique entourant le filtre et reliée à un électromètre. Elle permet, à travers la mesure du courant s'écoulant du filtre à la masse, de connaître la charge dans l'huile. Le filtre utilisé est une feuille de cellulose très poreuse, prévu pour une filtration de $3 \mu \mathrm{m}$; il est changé après chaque essai.

\section{Résultats expérimentaux - discussion.}

3.1 Essais paramétriques Des Couples Carton/Huile. Mesure du Courant moyen. L'étude des différents couples carton/huile montre que le courant moyen $I$ est peu affecté par l'huile, même si l'huile H2 semble créer légèrement plus de charges que H1 (Tab. I). En revanche, la nature du carton et son état de surface ont une influence remarquable. $I$ est plus élevé avec le carton $\mathrm{C} 2$ ( 3 à 6 fois) qu'avec $C 1$, et ce pour les deux types d'huile. La rugosité de la surface semble être à l'origine de cette différence. En effet, le carton C2 pré-compressé a

Tableau I.

\begin{tabular}{|c|c|c|}
\hline TYPE & TYPE & COURANTS MOYENS \\
DHULE & DE CARTON & ( PA) \\
\hline huile H1 & carton C1 & $-5,5$ \\
\hline huile $\mathrm{H} 1$ & carton $\mathrm{C} 2$ & $-27,7$ \\
\hline huile $\mathrm{H} 2$ & carton $\mathrm{Cl}$ & $-6,3$ \\
\hline huile $\mathrm{H} 2$ & carton $\mathrm{C} 2$ & $-30,3$ \\
\hline
\end{tabular}


une rugosité de surface de $100 \mu \mathrm{m}$ alors que celle de $\mathrm{Cl}$ est de $35 \mu \mathrm{m}$ (soit un rapport de l'ordre de 3).

Les courants créés par électrisation varient également avec la température et la vitesse de rotation du disque. les résultats obtenus en faisant varier la température de $20^{\circ} \mathrm{C}$ à $80^{\circ} \mathrm{C}$ et la vitesse de rotation de 80 à $160 \mathrm{tr} / \mathrm{min}$, confirment la tendance plus prononcée à l'électrisation du carton $\mathrm{C} 2$ par rapport à $\mathrm{C} 1$.

La caractéristique courant moyen-vitesse de rotation (Fig. 4), suit une fonction croissante quasi-linéaire de la forme :

$$
I(\mathrm{pA})=k \cdot V(\operatorname{tr} / \min )
$$

$k$ est une constante, comprise entre 0,15 et 0,5 , dépendant du couple carton-huile.

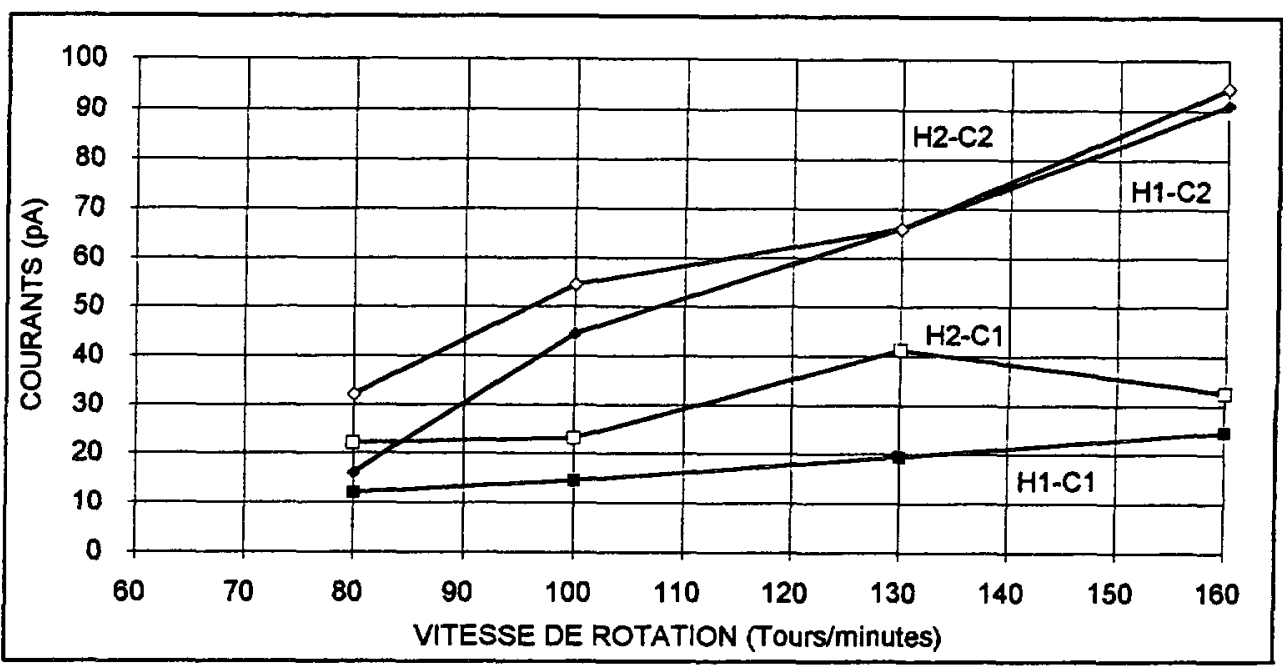

Fig. 4. - Evolution des courants en fonction de la vitesse de rotatıon dans le cas de la cellule CIGRE pour différents couples huile-carton.

[Currents is. rotating velocity in the case of ClGRE test cell for different oil-pressboard couples.]

Des essais complémentaires nous ont permis de confirmer cette linéarité pour des vitesses de rotation étendues à $430 \mathrm{tr} / \mathrm{min}$. Une telle linéairté apparaît souvent dans les études sur maquettes tubulaires pour des régimes d'écoulement laminaires [3, 8]. Pour ce même type de maquettes, la dépendance des courants et des densités de charges en fonction de la vitesse peut être plus que linéaire en régime turbulent $[3,9]$. L'augmentation du courant avec la vitesse, dans le cas des cellules d'essai à disque rotatif, a été également rapportée par d'autres chercheurs $[6,10]$; elle est attribuée à la composante normale de déplacement du fluide sur le disque.

En ce qui concerne l'influence de la température sur le courant (Fig. 5), elle est plus importante que celle de la vitesse de rotation du disque. Le cycle de montée en température est de 3 à $4 \mathrm{~h}$; la mesure des courants est effectuée lors d'une phase de déséquilibre hygrométrique entre l'huile et le carton. La croissance continue du courant avec la température se retrouve dans la mesure de la tendance à la charge des huiles et sur d'autres dispositifs expérimen$\operatorname{taux}[8,11]$. 


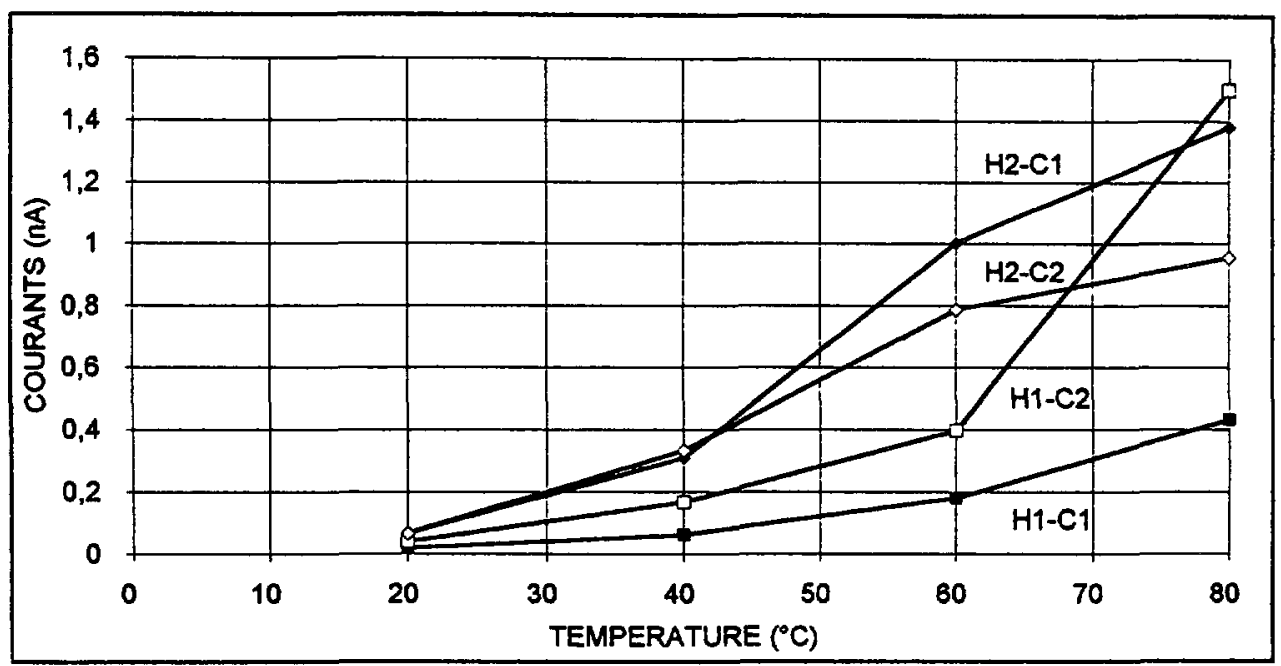

Fig. 5. - Evolution des courants en fonction de la température dans le cas de la cellule CIGRE pour différents couples huile-carton.

[Currents $v s$. temperature in the case of CIGRE test cell for different oil-pressboard couples.]

Le traitement des huiles (filtration et séchage) et celui des cartons (séchage) influent également sur la valeur des courants. Les résultats peuvent présenter des différences allant jusqu'à $50 \%$.

Quant à l'influence de la teneur en eau de l'huile sur les niveaux de courants, elle est relativement modérée lorsque le carton et l'huile sont à l'équilibre hygrométrique (Fig. 6), comme cela a été rapporté par Radwan et al. [12].

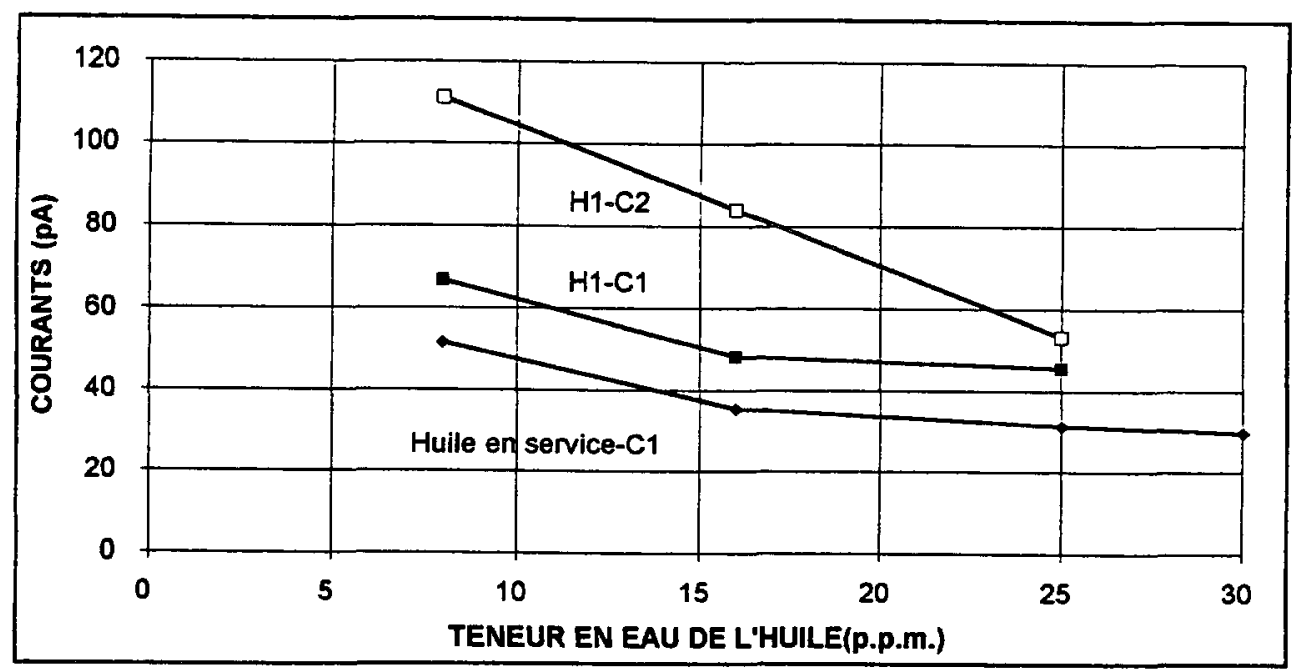

Fig. 6. - Influence de la teneur en eau de l'huile sur les niveaux de courants dans le cas de la cellule CIGRE pour différents couples huile-carton.

[Currents vs water content of oil in the case of CIGRE test cell for different oil-pressboard couples.] 
3.2 Mesure de la TEndance À la CHARge des huiles. - La tendance à la charge d'une huile a été étudiée en fonction de plusieurs paramètres.

3.2.1 Influence de la teneur en eau et de la température. - Pour étudier l'influence de la teneur en eau, nous avons testé deux huiles, avant et après reprise d'humidité à l'air libre : l'une à forte tendance à la charge (huile $\mathrm{H} 3$ ), l'autre à très faible tendance à la charge (huile H4). Nous remarquons (Tabl. II) que l'influence de la teneur en eau dans le cas de l'huile H4 est réduite. Cependant, les résultats sont discutables car pouvant être influencés par des paramètres non contrôlés ou par les incertitudes sur les mesures.

Tableau II.

\begin{tabular}{|l|c|c|}
\hline $\begin{array}{c}\text { TYPE } \\
\text { DHULLE }\end{array}$ & $\begin{array}{c}\text { TENEUR EN EAU } \\
\text { (p.p.m.) }\end{array}$ & $\begin{array}{c}\text { E.C.T. } \\
(\mu \mathrm{C} / \mathrm{m} 3)\end{array}$ \\
\hline huile neuve H3 sèche & 5 & 363 \\
\hline huile neuve H3 humide & 16 & 182 \\
\hline huile en service H4 sèche & 6 & 4 \\
\hline huile en service $\mathrm{H} 4$ humide & 13 & 15 \\
\hline
\end{tabular}

L'ensemble des mesures effectuées au laboratoire montre que les huiles à très forte teneur en eau ont généralement une faible tendance à la charge. Pour les teneurs en eau usuelles, la dispersion des points de mesure est très importante (Fig. 7). La siccité a plus d'importance visà-vis de la mesure dans le cas des huiles à forte E.C.T. du type H3. Des résultats similaires ont été obtenus par ailleurs [13,14].

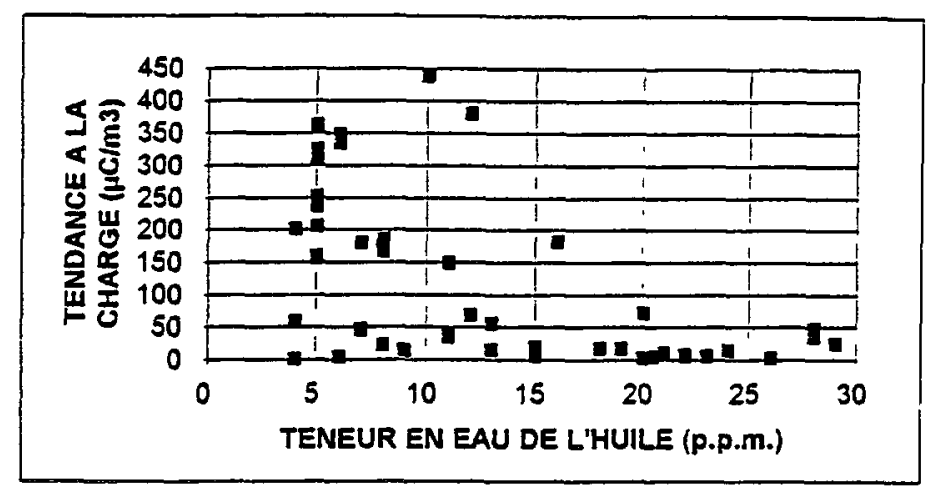

Fig. 7. - Influence de la teneur en eau sur la tendance à la charge des huiles minérales.

[Influence of the water content on the charging tendency of mineral oils.]

D'autre part, la densité de charge est très sensible à la présence d'humidité dans le filtre de l'appareil de mesure. Le tableau III montre l'importance de la teneur en eau avec des filtres non

Tableau III.

\begin{tabular}{|l|c|}
\hline \multicolumn{1}{|c|}{$\begin{array}{c}\text { TYPE } \\
\text { D'HUILE }\end{array}$} & $\begin{array}{c}\text { E.C.T. } \\
(\mu \mathrm{C} / \mathrm{m} 3)\end{array}$ \\
\hline huile H5 + filtre sec & 252 \\
\hline huile HS + filtre humide & 363 \\
\hline huile H6 + filtre sec & 325 \\
\hline huile H6 + filtre humide & 312 \\
\hline
\end{tabular}


séchés dont la teneur est de $4 \%$ à $6 \%$ et des filtres séchés à $1 \%$. Il apparaît donc prudent de faire des mesures avec une siccité contrôlée.

La tendance à la charge crô̂t également avec la température, alors que dans un transformateur, les densités de charge sont maximales pour une température de $40^{\circ} \mathrm{C}$ à $60{ }^{\circ} \mathrm{C}$. Une bonne reproductibilité des essais implique donc une température connue et constante.

3.2.2 Corrélation entre propriétés diélectriques et tendance à la charge. - Nous avons testé une cinquantaine d'huiles minérales d'origines diverses. Sur l'ensemble des huiles testées, il est possible de tracer les courbes de valeur de l'E.C.T. en fonction de la conductivité ou de l'angle de pertes diélectriques, comme le montre la figure 8 . Nous constatons que la tendance à la charge d'une huile est plus faible pour un faible angle de pertes, donc une faible conductivité. Cette constatation se vérifie particulièrement bien pour les huiles à très faibles E.C.T. Au-delà, la dispersion des points est plus grande. Des résultats similaires ont été obtenus par d'autres laboratoires [4, 15].

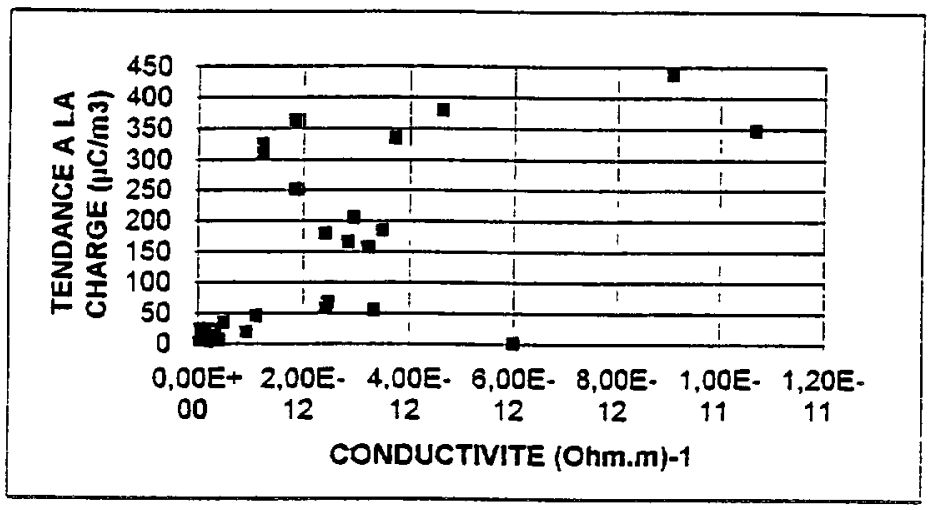

Fig. 8. - Tendance à la charge des huiles minérales en fonction de la conductivité.

[Charging tendency of mineral oils v's. conductivity.]

D'autre part, on constate que les huiles à forte tenue diélectrique sont celles qui ont une forte tendance à la charge, contraitement à ce à quoi on s'attendait au vu des résultats sur l'angle de pertes. La dispersion des points de mesure est ici plus faible (Fig. 9).

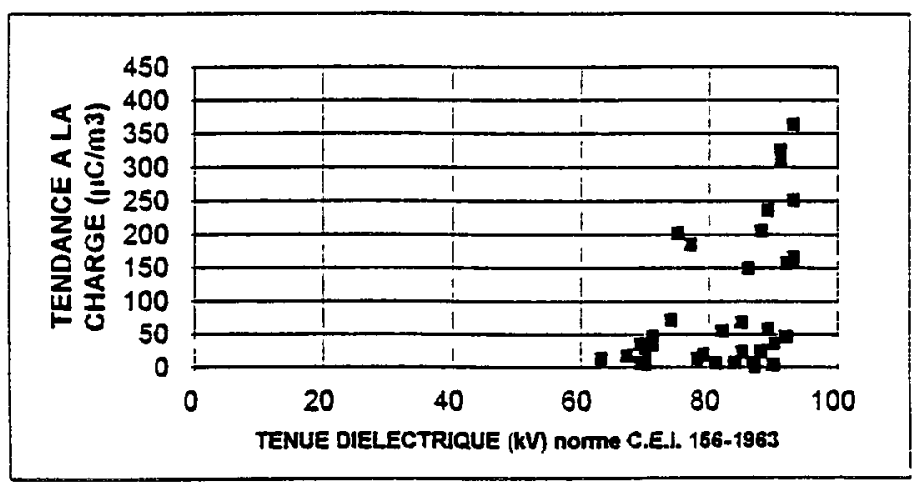

Fig. 9. - Corrélation entre la tendance à la charge des huiles et leur rigidité diélectrıque à $50 \mathrm{~Hz}$. [Correlation between the charging tendency of oils and their dielectric strength at $50 \mathrm{~Hz}$.] 
Les résultats obtenus montrent que les huiles vieillies [16], en service ou en stockage de longue durée, ont une E.C.T. plus forte. Le degré d'oxydation de l'huile est également un paramètre défavorable pour la tendance à la charge $[15,17]$. Ce paramètre est lié à l'angle de perte.

Nous avons également observé qu'une forte tendance à la charge est généralement accompagnée d'une faible tension interfaciale de l'huile, confirmant les résultats obtenus par ailleurs [13].

Remarquons qu'actuellement, il n'existe pas de consensus sur les types de « container » et de matériau à utiliser pour le prélèvement et le stockage des huiles $[17,18]$. Il nous semble néanmoins préférable d'utiliser le même type de container pour tous les essais, avec un temps de stockage le plus bref possible entre le prélèvement et les essais.

\section{Conclusion.}

Le mécanisme d'électrisation statique par circulation d'huile à travers les enroulements des transformateurs est un mécanisme très complexe. Son étude à travers des dispositifs expérimentaux à échelle réduite, a permis de mettre en évidence de nombreux paramètres tels que la température, la vitesse d'écoulement, l'humidité et la nature des interfaces.

En effet, la charge créée, mesurée sur la cellule de type CIGRE, augmente avec la rugosité du carton et la température. Elle croît de façon quasi-linéaire avec la vitesse. L'humidité de l'huile est un facteur de réduction de la charge. Cependant, si les résultats obtenus sont très instructifs, l'influence des migrations de l'eau entre carton et huile, des gradients de température et des particules demandent à être clarifiée.

La mesure de la tendance à la charge des huiles semble être une indication précieuse pour évaluer le niveau de densité de charges susceptibles d'être atteint par une huile en service. Une corrélation peut être établie entre cette grandeur et les caractéristiques physico-chimiques et diélectriques de l'huile minérale. Ainsi, les fortes tendances à la charge sont généralement corrélées à de faibles teneurs en eau, à de fortes rigidités diélectriques, à des tensions interfaciales peu élevées et à de grands angles de pertes.

\section{Bibliographie}

[1] Takagi T., Okada T., Kurita K., Tamura R., Murata H., Amélioration de la fiabilité des transformateurs $500 \mathrm{kV}$ de grande puissance, CIGRE (Session 1978) W.G. 12-02.

[2] Zahn M., Electrical breakdown in transformers due to flow electrification, publication privée.

[3] Oomen T. V., Lindgren S. R., Streaming electrification study of transformer insulation system using a paper tube model, I.E.E.E. Trans. Pow'er Delivry 5 (1990) 972.

[4] Shimizu S., Murata H., Honda M., Electrostatics in power transformers, I.E.E.E. Trans. Power Apparatus Syst. 98 (1979) 1244.

[5] Praxi G., Chobel W., Screening of material focused on electrification of solid/oil interfaces used in power transformers, CIGRE (Session 1990) S.C. 12/15, J.W.G. 13, T.F. 01.

[6] Kedzia J., Investigation of transformer oil electrification in a spinning disk system, I.E.E.E. Trans. Electr. Insul. 24 (1989) 59.

[7] Oommen T. V., Static electrification properties of transformer oil, I E.E.E. Trans. Electr. Insul. 23 (1988) 123.

[8] Moser H. P., Krause Ch., Pralx G., Spandonis G., Stonitsch, Electrostatic charging in large size models of power transformer cooling ducts CIGRE (Session 1992) J.W.G. 12/15.13. T.F. 01T.F. 02 . 
[9] Higaki M., Kako Y., Moriyama M., Hirano M., Hiraishi K., Kurita K., Static electrification and partial discharges caused by oil flow in forced oil cooled core type transformers, I E.E.E. Trans. Power Appar. Syst. 98 (1979) 1259.

[10] Praxl G., Lemesh G., Measurement of ECT with the CIGRE test cell, CIGRE (Session 1993) J.W.G. $12 / 15-13$.

[11] Lindgren S. R., Wasabaugh A. P., Zahn M., Brubaker M., Nelson J. K., Von Guggenberg P., Temperature and moisture transient effects on flow electrification in power transformers, CIGRE (Session 1992) J.W.G. 15/12-02.

[12] Radwan R. M., El-Dewieny R. M., Metwally I. A., Investigation of static electrification phenomenon due to transformer oil flow in electric power apparatus, I.E.E.E Trans. Electr. Insul. 27 (1992) 1275.

[13] Oommen T. V., Petrie E. M., Electrostatic charging tendency of transformer oils, I.E.E.E. Trans. Pow'er Appar. Syst. 103 (1984) 1923.

[14] Okubo H., Ikeda M., Honda M., Yanari T., Charging tendency measurement of transformer oil, I.E.E.E. Power Engineering Society, Winter meeting (February 1979).

[15] Tamura R., Miura Y., Watanabe T., Ishii T., Yamada N., Nitta T., Statıc electrification by forced oil flow in large power transformer, I.E.E.E. Trans. Power Appar. Syst. 99 (1980) 335.

[16] Yasuda M., Goto K., Okubo H., Ishii T., Mori E., Masunaga M., Suppression of static electrification of insulating oil for large power transformers, I.E.E.E. Trans. Pow'er Appar. Syst. 101 (1982) 4272.

[17] Griffin P. J., Electrostatic Charging Tendency of transformer oils, DOBLE Engineenng Company, rapport interne Sec. 6-1201 (1985).

[18] Reygaerts A., Static electrification of mineral oil used in power transformers, rapport interne A.R. Rapelst1-co2/099510, LABORELEC (1993). 